\title{
Einbezug der Stakeholder bei der Beurteilung von Handlungsfeldern der gesellschaftlichen Verantwortung nach ISO 26000 in kleinen und mittleren Unternehmen
}

\author{
Mariana Christen Jakob $\cdot$ Herbert Winistörfer
}

Online publiziert: 8. April 2013

(C) Springer-Verlag Berlin Heidelberg 2013

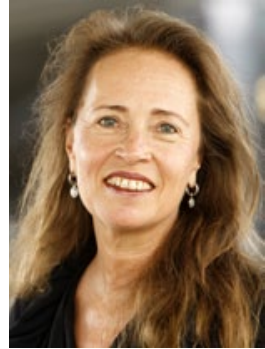

M. Christen Jakob

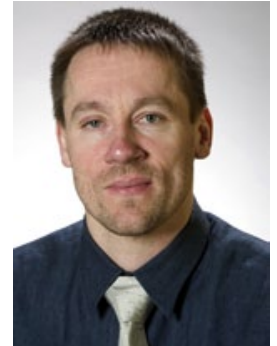

H. Winistörfer

Zusammenfassung Verschiedene Untersuchungen zeigen auf, dass Corporate Social Responsibility (CSR) bei kleinen und mittleren Unternehmen (KMU) mit spezifischen Konzepten und Methoden anzugehen ist. Im Hinblick auf eine systematische und global gültige Umsetzung wurde die Norm ISO 26000 zur gesellschaftlichen Verantwortung entwickelt, der u. a. eine stärkere Verbreitung unter den KMU erreichen möchte. Eine der sieben Grundsätze der Norm fordert bei der Implementierung den Einbezug der Anspruchsgruppen, unter anderem bei der Auswahl der zu bearbeitenden Handlungsfelder. Bei der Implementierung in die Unternehmenspraxis stellen sich für KMU nicht zu unterschätzende Herausforderungen. Im Rahmen eines Forschungsprojekts wurden vier KMU bei diesem Implementierungsprozess begleitet. Im Vordergrund stand die Frage, welche unterstützenden Instrumente und Methoden

M. Christen Jakob $(\bowtie)$

Hochschule Luzern - Soziale Arbeit, Werftestrasse 1,

Postfach 2945, 6002 Luzern, Schweiz

E-Mail: mariana.christen@hslu.ch

H. Winistörfer

ZHAW School of Management and Law, Postfach 805, 8401 Winterthur, Schweiz

E-Mail:winh@zhaw.ch

für eine optimale Lösung dieser Herausforderungen nötig sind. Erste Ergebnisse im Hinblick auf den Einbezug von Stakeholdern zeigen, dass der Nutzen trotz anfänglichen Vorbehaltes seitens der Unternehmen als hoch eingestuft wurde und zwar gerade von Unternehmen, die aufwändige Formen gewählt haben. Bei der Wahl der Methoden haben sich durchweg Formen des persönlichen Austausches mit einer eher geringen Anzahl von Personen gegenüber breit angelegten schriftlichen Befragungen durchgesetzt. Eine große Herausforderung bestand darin, die Handlungsfelder von ISO 26000 den befragten Stakeholdern so verständlich zu machen, dass eine Gewichtung möglich wurde.

\section{Gesellschaftliche Verantwortung und KMU}

Dass Unternehmen, auch KMU, Mitverantwortung für die Lösung gesellschaftlicher Probleme über die gesetzlichen Mindestanforderungen hinaus freiwillig übernehmen, ist kein neues Phänomen. Es lässt sich auf das mittelalterliche Konzept des ,ehrbaren Kaufmanns“ zurückführen (vgl. Klink 2008). In neuerer Zeit verwenden Unternehmen die Begriffe unternehmerische Nachhaltigkeit (Corporate Sustainability), bürgerschaftliches Engagement (Corporate Citizenship) oder gesellschaftliche Verantwortung (Corporate Social Responsibility, CSR) Das letztgenannte Konzept hat seinen Ursprung im angloamerikanischen Raum und gewinnt im Zuge der fortschreitenden Globalisierung der Wertschöpfungsketten weite Verbreitung. Vor allem in großen multinational tätigen Unternehmen (MNU) ist es üblich geworden, eine Reihe von Aktivitäten unter den genannten Begriffen zu führen und zwar von der Formulierung von Leitbildern, über die Führung von Fach- oder Stabsstellen bis zur Veröffentlichung entsprechender Rechenschaftsberichte als Ergänzung zur Finanzberichterstattung (vgl. 
Daub et al. 2007). Bei kleinen und mittleren Unternehmen sind diese Ansätze noch wenig geläufig, obwohl es starke Hinweise dafür gibt, dass sie auch für KMU nutzenstiftend sein und die Wettbewerbsfähigkeit steigern können (vgl. Castka et al. 2004; Enderle 2004). Allerdings zeigen jüngere Untersuchungen (Murillo und Lozano 2006; Perrini und Minoja 2008; Spence 1999; Spence et al. 2003; Vyakarnam et al. 1997), dass die tatsächlich erbrachten gesellschaftlichen Leistungen von KMU empirisch durchaus belegt werden können. Unternehmen engagieren sich häufig in unterschiedlichsten Handlungsfeldern intern und extern, sind aber mit den Hintergründen des CSR-Ansatzes weniger vertraut und gehen die Umsetzung weniger systematisch und strategisch an (vgl. Eichholz 2007, S. 12; Christen Jakob und von Passavant 2009). Die wissenschaftliche Diskussion (Murillo und Lozano 2006; Perrini und Minoja 2008) zeigt denn auch deutlich auf, dass es für den KMU-Sektor spezifische Konzepte und Instrumente braucht, welche den besonderen Umständen und Bedürfnissen der KMU Rechnung tragen. Typische Merkmale der KMU-Landschaft wie flache Hierarchien, geringer Grad der Formalisierung, Multifunktionalität in den Aufgabenfeldern, fehlende interne Verfügbarkeit von CSR-spezifischem Fachwissen oder Knappheit an finanziellen und zeitlichen Ressourcen (vgl. Lepoutre und Heene 2006, 263 ff.; Jenkins 2004) gilt es insbesondere bei Fragen der Implementierung eines systematischen und strategischen CSR-Ansatzes zu beachten.

Anfang 2000 hat die Europäische Kommission die Idee der gesellschaftlichen Verantwortung von Unternehmen aufgegriffen und im Grünbuch folgende Definition festgehalten (Europäische Kommission 2001, S. 7): „CSR ist ein Konzept, das den Unternehmen als Grundlage dient, auf freiwilliger Basis soziale Belange und Umweltbelange in ihre Tätigkeit und in die Wechselbeziehungen mit den Stakeholdern zu integrieren." Verschiedene Elemente sind in dem hier definierten CSR-Konzept von besonderer Bedeutung:

- Das unternehmerische Engagement ist freiwillig und geht über die gesetzlichen Vorschriften hinaus;

- CSR umfasst betriebswirtschaftliche, soziale und ökologische Dimensionen;

- CSR ist integrierter Teil der Geschäftstätigkeit (und nicht lediglich eine gut gemeinte Begleitaufgabe);

- CSR ist mit dem Stakeholderansatz verknüpft.

In einer überarbeiteten Version der neuen EU-Strategie (2011-2014) für die soziale Verantwortung der Unternehmen (Europäische Kommission 2011) werden alle großen europäischen Unternehmen aufgefordert, sich bis 2014 entweder zu den Leitsätzen des UN Global Compact oder zur internationalen Norm ISO $26000 \mathrm{zu}$ verpflichten. Damit ist absehbar, dass sich auch für KMU, besonders für jene, die in eine europäische oder internationale Wertschöpfungskette eingebunden sind, der Druck erhöht, eine CSR-Strate- gie konzeptionell anzugehen und diese entsprechend $\mathrm{zu}$ implementieren.

\section{Die internationale Norm ISO 26000}

Vor dem Hintergrund einer Vielzahl von Definitionen, Konzepten und Richtlinien zur gesellschaftlichen Verantwortung von Organisationen wollte die Internationale Organisation für Normung ISO mit einem umfassenden Konzept richtungsweisende Anleitungen für diese Aufgabe vorgeben. Das ist deshalb folgerichtig, weil die ISO in verwandten Themenbereichen wie Qualitätsmanagementsysteme (ISO 9000) oder Umweltmanagementsysteme (ISO 14001) erfolgreiche Normen entwickelt hat. Damit verfügt die ISO über die nötigen Strukturen und Prozesse, um in einem mehrjährigen Prozess einen internationalen Konsens zu einem komplexen und kontrovers diskutierten Gegenstand zu erreichen. Die Norm ISO 26000 (SNV 2011) ist in diesem Sinne eine logische Weiterentwicklung mit dem Ziel, einen umfassenden Rahmen zum Umgang mit gesellschaftlich relevanten Themen zu geben, ohne die bestehenden, themenspezifischen Normen zu ersetzen. Im Unterschied zu jenen ist die neue Norm nicht als zertifizierbarer Katalog von überprüfbaren Anforderungen konzipiert, sondern als „Internationale Norm mit Leitfadencharakter" (SNV 2011, S. 13).

Die am 1. November 2010 publizierte Norm ISO 26000 zu „Social Responsibility“ (SR) wurde während mehr als sechs Jahren von Fachleuten aus über 90 Ländern in einem großen Multi-Stakeholder-Prozess entwickelt (vgl. Lang und Winistörfer 2010). Die Norm nimmt alle bereits im EU-Grünbuch formulierten Aspekte von CSR auf (Freiwilligkeit, Integration in die Kernprozesse, Stakeholderansatz) und umfasst sieben Abschnitte und einen informativen Anhang. Den materiellen Hauptteil bildet der sechste Abschnitt. Er definiert sieben Kernthemen und in zweiter Ordnung 37 Handlungsfelder der gesellschaftlichen Verantwortung von Organisationen. Dazu wird jeweils im Detail beschrieben, wie die Handlungsfelder zu verstehen sind und welche Massnahmen von Organisationen erwartet werden können. Tabelle 1 zeigt die sieben Kernthemen mit den dazugehörigen Handlungsfeldern.

\section{Die Bedeutung des Stakeholderdialogs}

In der wissenschaftlichen wie der praxisorientierten Literatur herrscht weitgehend Einigkeit darüber, dass die Bedeutung einer systematischen Auseinandersetzung mit Stakeholdern für Unternehmen zunimmt (vgl. Burchell und Cook 2008; Camillus 2008; Crane und Livesey 2003; Freeman et al. 2007; Sachs et al. 2007; Yaziji und Doh 2009). Als Gründe dafür werden verschiedene Entwicklungen genannt: 
Tab. 1 Kernthemen und Handlungsfelder aus ISO 26000 (SNV 2011, S. 10 f.)

\begin{tabular}{|c|c|}
\hline Kernthemen & Handlungsfelder \\
\hline Organisationsführung & Prozesse und Strukturen der Entscheidungsfindung \\
\hline Menschenrechte & $\begin{array}{l}\text { Gebührende Sorgfalt; Menschenrechte in kritischen Situationen; Mittäterschaft vermeiden; Missstände beseitigen; } \\
\text { Diskriminierung und schutzbedürftige Gruppen; Bürgerliche und politische Rechte; Wirtschaftliche, soziale und } \\
\text { kulturelle Rechte; Grundlegende Prinzipien und Rechte bei der Arbeit }\end{array}$ \\
\hline Arbeitspraktiken & $\begin{array}{l}\text { Beschäftigung und Beschäftigungsverhältnisse; Arbeitsbedingungen und Sozialschutz; Sozialer Dialog; Gesundheit } \\
\text { und Sicherheit am Arbeitsplatz; Menschliche Entwicklung und Schulung am Arbeitsplatz }\end{array}$ \\
\hline Umwelt & $\begin{array}{l}\text { Vermeidung der Umweltbelastung; Nachhaltige Nutzung von Ressourcen; Abschwächung des Klimawandels und } \\
\text { Anpassung; Umweltschutz, Artenvielfalt und Wiederherstellung natürlicher Lebensräume }\end{array}$ \\
\hline $\begin{array}{l}\text { Faire Betriebs- und } \\
\text { Geschäftspraktiken }\end{array}$ & $\begin{array}{l}\text { Korruptionsbekämpfung; Verantwortungsbewusste politische Mitwirkung; Fairer Wettbewerb; Gesellschaftliche } \\
\text { Verantwortung in der Wertschöpfungskette fördern; Eigentumsrechte achten }\end{array}$ \\
\hline Konsumentenanliegen & $\begin{array}{l}\text { Faire Werbe-, Vertriebs- und Vertragspraktiken sowie sachliche und unverfälschte, nicht irreführende Informationen; } \\
\text { Schutz von Gesundheit und Sicherheit der Konsumenten; Nachhaltiger Konsum; Kundendienst, Beschwerdema- } \\
\text { nagement- und Schlichtungsverfahren; Schutz und Vertraulichkeit von Kundendaten; Sicherung der Grundversor- } \\
\text { gung; Verbraucherbildung und Sensibilisierung }\end{array}$ \\
\hline $\begin{array}{l}\text { Einbindung und } \\
\text { Entwicklung der } \\
\text { Gemeinschaft }\end{array}$ & $\begin{array}{l}\text { Einbindung der Gemeinschaft; Bildung und Kultur; Schaffung von Arbeitsplätzen und berufliche Qualifizierung; } \\
\text { Technologien entwickeln und Zugang dazu ermöglichen; Schaffung von Wohlstand und Einkommen; Gesundheit; } \\
\text { Investition zugunsten des Gemeinwohls }\end{array}$ \\
\hline
\end{tabular}

Zum einen werden die Wertschöpfungsketten zunehmend global vernetzt. Dadurch wird die Stakeholderlandschaft für Unternehmen komplexer, sowohl was marktliche Stakeholder (z. B. Kunden, Lieferanten) betrifft als auch nicht-marktliche (z. B. Behörden, Nochtregierungsorganisationen). Parallel dazu steigt die Anzahl zivilgesellschaftlicher Akteure, welche die Aktivitäten von Unternehmen genau beobachten. Auch sie agieren zunehmend global und sind untereinander vernetzt. Ihre Tätigkeit wird erleichtert durch die modernen Informations- und Kommunikationstechnologien. Dadurch nimmt die Sichtbarkeit der Unternehmen zu (gläserne Unternehmen). Mit steigendem Wohlstand, auch in Schwellenländern wie China, steigen die Erwartungen an Unternehmen - sogenannte Wohlstands-Themen wie „Umweltschutz“ oder „Arbeitsschutz“, die häufig durch komplexe Ursache-Wirkungsbeziehungen geprägt sind, gewinnen so an Bedeutung. Insgesamt wird so die gesellschaftliche Betriebsbewilligung (,licence to operate") immer wichtiger, die massgeblich von der Wahrnehmung der Unternehmen durch deren Stakeholder abhängig ist.

Der Nutzen einer systematischen Auseinandersetzung mit den Stakeholdern und deren Erwartungen wird für Unternehmen unter anderem in folgenden Aspekten gesehen (vgl. Winistörfer et al. 2012, S. 20):

- Relevante Themen sowie deren Chancen und Risiken für das Unternehmen frühzeitig erkennen;

- Höhere Akzeptanz von Entscheiden und Handlungen erreichen durch Einbindung der Stakeholder in die Lösungsentwicklung;

- Betriebsblindheit überwinden durch Integration verschiedener Sichtweisen;
- Besseres Verständnis dafür entwickeln, wie sich die unternehmerischen Produkte, Entscheidungen und Aktivitäten auf die;

- verschiedenen Stakeholder und die Gesellschaft insgesamt auswirken;

- Partnerschaften und Kooperationen aufbauen für Knowhow-Transfer oder die gemeinsame Bearbeitung komplexer Probleme, die im Alleingang nicht gelöst werden können;

- Vertrauen aufbauen für transparente und tragfähige Beziehungen.

Es ist deshalb folgerichtig, dass sämtliche modernen Ansätze zum Umgang von Unternehmen mit gesellschaftlicher Verantwortung ${ }^{1}$ dem Dialog mit und dem Einbezug von Stakeholdern grosse Bedeutung zumessen.

ISO 26000 thematisiert die Stakeholder unter mehreren Gesichtspunkten. In einem einleitenden Kapitel wird die „Rolle der Anspruchsgruppen im Kontext gesellschaftlicher Verantwortung" als eines von mehreren Merkmalen gesellschaftlicher Verantwortung thematisiert (vgl. ISO 26000, Kap. 3.3.3). Dann wird die „Achtung der Interessen von Anspruchsgruppen“ als einer von sieben Grundsätzen gesellschaftlicher Verantwortung beschrieben (vgl. ISO 26000, Kap. 4.5). Schließlich wird die „Identifizierung und Einbindung der Anspruchsgruppen" als eine von zwei fundamentalen Praktiken neben der „Anerkennung gesellschaftlicher Verantwortung “ bei der Umsetzung von gesellschaftlicher Verantwortung eingeführt (vgl. ISO 26000, Kap. 5.3).

\footnotetext{
${ }^{1}$ Vgl. zum Beispiel: OECD-Leitsätze für multinationale Unternehmen, Global Reporting Initiative, ISO 26000.
} 


\section{Umsetzungshilfen für KMU als Ziel eines Forschungsprojekts}

Typischerweise setzen sich KMU - obwohl sie häufig andere Begriffe verwenden - mit ausgewählten Stakeholdern auseinander und bearbeiten auch einzelne CSRHandlungsfelder. Allerdings findet eine systematische Auseinandersetzung mit Stakeholdern wie auch, darauf aufbauend, eine systematische und strategisch orientierte Umsetzung von CSR bisher selten statt (Jamali et al. 2009). Dafür bietet die Handlungsfelderstruktur von ISO 26000 eine gute Grundlage. Für Organisationen, die die Norm anwenden wollen, gerade für KMU, stellen sich jedoch einige zentrale Herausforderungen: die Struktur der Norm ist komplex, der Text umfangreich, die Sprache anspruchsvoll und es braucht zusätzlich eine Anleitung, wie die Normeninhalte Schritt für Schritt umgesetzt werden können. Der Anwendungsbereich der Norm ist bewusst sehr breit gehalten: Angesprochen sind alle Arten von Organisationen unabhängig von der Branche, der Größe und dem Standort. Auf die spezielle Situation von KMU wird lediglich in einem kurzen Abschnitt eingegangen (vgl. ISO 26000, Kap. 3.3.4). Hier setzt ein anwendungsorientiertes Forschungsprojekt der Hochschule Luzern HSLU und der Zürcher Hochschule für Angewandte Wissenschaften ZHAW an. Im Zentrum steht die Frage, ob und wie genau die Struktur der Handlungsfelder aus ISO 26000 genutzt werden kann, um den CSR-Ansatz von KMU strategisch und systematisch auszurichten. Dafür wird auf der Basis der bestehenden Literatur ein schrittweises Vorgehen zusammen mit konkreten Instrumenten entworfen und anschlieBend in Zusammenarbeit mit vier Unternehmen in der Praxis erprobt. Diese Pilotprojekte werden evaluiert, und anhand der gewonnenen Erfahrungen werden Vorgehensweise und Instrumente überarbeitet und so aufbereitet, dass sie von Unternehmen selbstständig angewendet werden können. Ziel ist die Erarbeitung eines Prozesshandbuchs, basierend auf Praxiserfahrungen, das KMU bei der Anwendung von ISO 26000 unterstützen kann.

\section{Die am Pilotprojekt teilnehmenden Unternehmen}

Für das Pilotprojekt wurden Unternehmen gesucht, die dem Segment der KMU zugeordnet werden können, üblicherweise charakterisiert durch eine Mitarbeitendenzahl bis 250 . Die Unternehmen sollten divers sein bezüglich Branchenzugehörigkeit, Lieferantenstruktur, Marktstruktur und damit auch bezüglich ihrer Exposition gegenüber verschiedenen Handlungsfeldern der gesellschaftlichen Verantwortung. Sie sollten mindestens erste Ansätze einer strukturierten und systematischen Auseinandersetzung mit CSR aufweisen und als ausdrückliches Ziel diese weiter entwickeln wollen. Vier KMU nehmen am Pilotprojekt teil:

- Unternehmen A: Ein traditionelles Familienunternehmen in der Herstellung von Haushaltgeräten; ca. 160 Mitarbeitende; wichtigen Lieferanten aus Fernost und weltweites Vertriebsnetz; nach ISO 9001 zertifiziertes Managementsystem und ausgezeichnetem Gesundheitsmanagement (Friendly Work Space ${ }^{\circledR}$ ).

- Unternehmen B: Ein industrieller Bäckereibetrieb im Privatbesitz; ca. 50 Mitarbeitende; Herkunft der Zulieferprodukte fast ausschließlich in Europa; ca. 10\% Exportanteil, zertifiziertes Managementsystem nach ISO 9001, ISO 14001 und ISO 31000 (Risikomanagement).

- Unternehmen C: Eine genossenschaftlich organisierte Regionalbank mit starker lokaler Verankerung und Schwerpunkt im Kreditgeschäft mit Privat- und Unternehmenskunden; ca. 65 Mitarbeitende; Managementansatz nach EFQM (European Foundation for Quality Management) mit externem Assessment.

- Unternehmen D: Hersteller optischer Produkte für vorwiegend den Schweizer Markt mit fast 100jähriger Familientradition; ca. 55 Mitarbeitende; regelmäßige, mehrfach ausgezeichnete Berichterstattung zu Umwelt und Nachhaltigkeit seit 1997.

\section{Ein Modell des Vorgehens in mehreren Phasen}

Die Annäherung an die Handlungsfelder von ISO 26000 erfolgte im Pilotprojekt in mehreren Phasen (vgl. Abb. 1). Zunächst erfolgte eine Analyse über alle 37 Handlungsfelder entlang von drei Perspektiven (Phase 1). Darauf aufbauend wurden einige wenige Handlungsfelder für die Weiterentwicklung ausgewählt (Phase 2). Dann wurden für jedes der ausgewählten Handlungsfelder die Ziele der Weiterentwicklung im Einklang mit der Unternehmensstrategie bestimmt und es wurden entsprechende Projekte und Maßnahmen geplant (Phase 3). Daraufhin wurden die geplanten Projekte realisiert (Phase 4). Dabei wurde darauf geachtet, dass die vorgenommenen Änderungen dauerhaft im Managementprozess verankert und mit den bestehenden Instrumenten der Unternehmensführung verknüpft werden (Phase 4). Mit genügend zeitlicher Distanz erfolgt eine neue Analyse (Phase 1), die den Fortschritt dokumentiert. Dieser Zyklus wird in der vom Unternehmen gewählten Periodizität durchlaufen.

\section{Eine dreistufige Analyse liefert die Entscheidungsgrundlagen}

Laut Lepoutre und Heene (2006, S. 267) liegt ein wesentliches Hindernis für KMU, im Bereich der gesellschaftlichen 
Abb. 1 Modell des schrittweisen Vorgehens für eine systematische und strategische Implementierung der SR-Handlungsfelder nach ISO 26000. (Quelle: Angelehnt an Lang et al. 2012, S. 24)

Abb. 2 Kombination von drei Perspektiven zur Festlegung von Handlungsschwerpunkten. (Quelle: Angelehnt an Lang et al. 2012, S. 25)

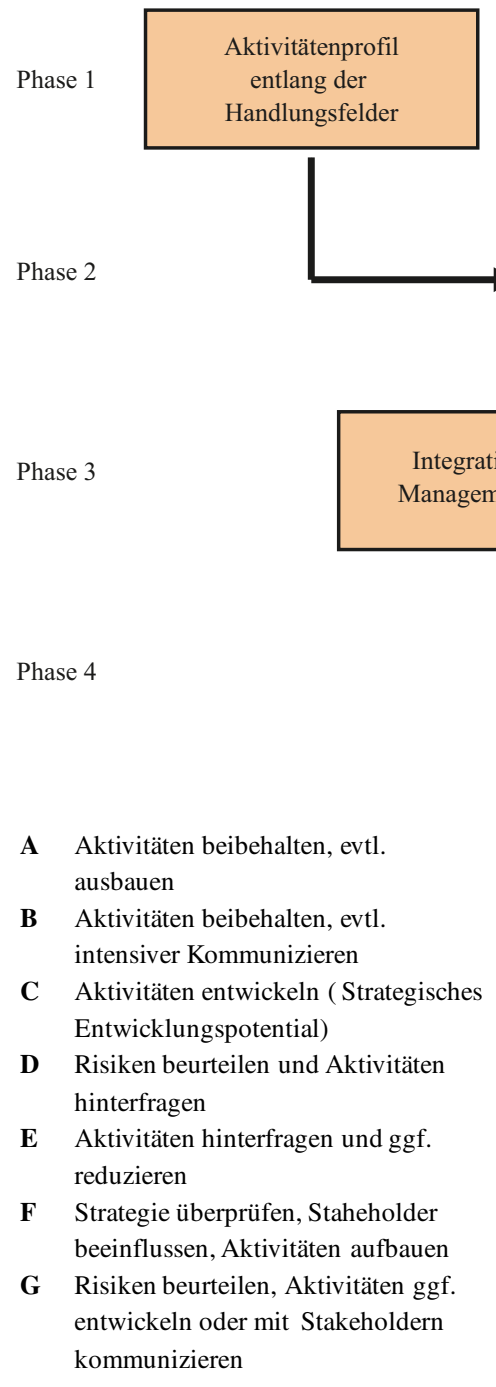

A Aktivitäten beibehalten, evtl. ausbauen

Aktivitäten beibehalten, evtl er Kommuniziere

Aktivitäten entwickeln ( Strategisches

Risiken beurteilen und Aktivitäten

reduzieren

beeinflussen, Aktivitäten aufbauen kommunizieren

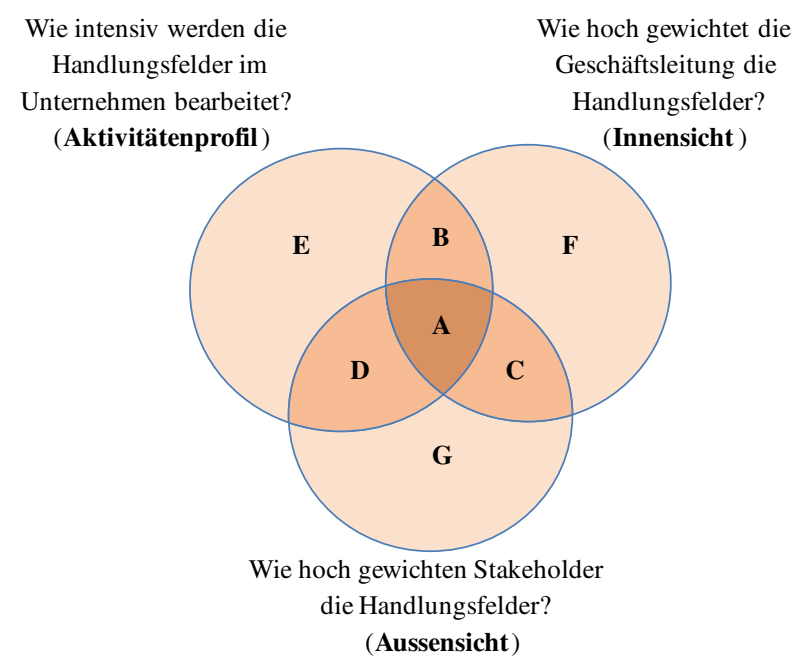

Verantwortung aktiv zu sein darin, dass sie Handlungsfelder häufig nicht als solche erkennen. Andererseits sind Gerade KMU angesichts der Vielzahl möglicher Handlungsfelder überfordert. Zudem ist es unmöglich, mit den knappen Ressourcen viele Handlungsfelder gleichzeitig weiterzuentwickeln. Der Analyse in der ersten Phase kommt große Bedeutung $\mathrm{zu}$, indem es darum geht, Handlungsfelder $\mathrm{zu}$ erkennen und zu priorisieren. Dies geschieht auf der Basis einer Analyse mit drei Perspektiven (vgl. Abb. 2). Für eine erste Perspektive wurde vom Unternehmen selbst ein auf dem Normentext basierender Fragenkatalog zu allen 37 Handlungsfeldern bearbeitet. Dabei wurden in Form einer Bestandsaufnahme alle Unternehmensaktivitäten erfasst und dokumentiert, die sich den Handlungsfeldern von ISO 26000 zuordnen lassen. Gleichzeitig wurde anhand einer Skala für jedes Handlungsfeld beurteilt, wie weit die von der Norm formulierten Vorgaben erfüllt sind. So entsteht ein Aktivitätenprofil als Standortbestimmung. Für die zweite Perspektive wurden alle 37 Handlungsfelder danach beurteilt, welche Bedeutung sie aus Sicht der Geschäftsleitung für das Erreichen der Unternehmensziele haben. Das geschah in Form eines moderierten Workshops unter Einbezug verschiedener leitender Führungskräfte. Auch hier wurde mit einer mehrstufigen Skala gearbeitet, die es erlaubte, den Handlungsfeldern als Ergebnis eines Einigungsprozesses ein relatives Gewicht als Innensicht des Unternehmens zuzuordnen.

Für die dritte Perspektive wurden ausgewählte Stakeholder danach gefragt, welche Bedeutung sie den einzelnen Handlungsfeldern im Zusammenhang mit dem betreffenden Unternehmen zuordnen. Hier diente die gleiche Skala der Zuordnung relativer Gewichte zu den Handlungsfeldern als Aussensicht. Innensicht und Aussensicht entsprechen dabei den beiden Kriterien, die auch die Global Reporting Initiative vorschlägt, um die Materialität der CSR-Themen im Unternehmen zu bestimmen (vgl. GRI 2011, S. 8). Erst die 
Kombination aller drei Perspektiven auf die Handlungsfelder ermöglicht es, strategische Schwerpunkte zu setzen und eine Auswahl jener Handlungsfelder zu treffen, in die Ressourcen für eine Weiterentwicklung investiert werden. Wird ein Handlungsfeld beispielsweise sowohl in der Innen- wie der Aussensicht hoch gewichtet, ist aber nicht durch entsprechende Aktivitäten abgedeckt (vgl. Abb. 2, Bereich C), liegt es nahe, dieses Handlungsfeld weiterzuentwickeln. Für den Fall, dass ein intern für wichtig befundenes und durch Aktivitäten gut abgedecktes Handlungsfeld von den Stakeholdern für wenig wichtig eingestuft wird (vgl. Abb. 2, Bereich B), gilt es die Kommunikation zu den entsprechenden Stakeholdern zu überprüfen.

Im Folgenden wird die Gewichtung der Handlungsfelder durch die Stakeholder (Aussensicht) am Beispiel der vier am Pilotprojekt beteiligten KMU dargestellt. Basis dazu bilden die Auswertungen von Fokusgruppen-Meetings mit allen im Projekt beteiligten Partnern ebenso wie die Beobachtungen in Stakeholderworkshops der verschiedenen KMU. Ergänzend dazu wurden Experteninterviews zum Thema „Stakeholderdialog im Rahmen des CSRProzesses" mit den internen Projektleitern der vier KMU durchgeführt, aufgezeichnet, transkribiert und qualitativ ausgewertet.

\section{Unterschiedliche Vorgehensweisen für den Einbezug der Stakeholder}

Über die Bestimmung der Wesentlichkeit der verschiedenen Handlungsfelder sagt die Norm ISO 26000 (Kap. 7.3.2.2): „[Die Organisation sollte] einen Bewertungsmaßstab entwickeln, mit dessen Hilfe entschieden werden kann, welche Handlungsfelder die größte Bedeutung für die Organisation haben. Mögliche Bewertungskriterien beinhalten:

- wie groß die mit einem Handlungsfeld verbundenen Auswirkungen auf die Anspruchsgruppen oder auf nachhaltige Entwicklung sind; [...]

- wie stark die Bedenken der Anspruchsgruppen in Bezug auf ein Handlungsfeld sind; und

- welche Erwartungen die Gesellschaft an verantwortliches Verhalten im Umgang mit diesen Auswirkungen hat."

Im Pilotprojekt wird den skizzierten Kriterien mit der oben beschriebenen Perspektive der „Aussensicht“ entsprochen. Dies geschah in drei Schritten: Zunächst waren diejenigen Stakeholdergruppen ausgewählt, deren Meinung eingeholt werden sollte; dann wurde die Methode des Einbezugs definiert und schließlich die $\mathrm{zu}$ befragenden Personen identifiziert.

Die Tab. 2 zeigt auf, welche Wege von den Unternehmen im Pilotprojekt gewählt wurden.

\section{Erkenntnisse zur Auswahl der Stakeholder}

Damit im Sinne von ISO 26000 ein Stakeholder als legitim gilt, müssen verschiedene Kriterien erfüllt sein (Hardtke und Kleinfeld 2010, S. 29): Die potenziellen Anspruchsgruppen müssen von den Folgen der unternehmerischen Aktivitäten betroffen sein, im Weiteren müssen auch die vorgebrachten Interessen selbst mit den Grundsätzen der gesellschaftlichen Verantwortung bzw. der nachhaltigen Entwicklung vereinbar sein Selbst mit dieser inhaltlichen Eingrenzung stehen die Unternehmen bei der Auswahl der Stakeholder für eine Befragung vor nicht zu unterschätzenden Herausforderungen. KMU verfügen über knappe zeitliche und finanzielle Ressourcen für eine Stakeholderbefragung. „Wenn man es richtig machen will, ist es arbeitsaufwändig. Das könnte ein Grund sein, weshalb gewisse Unternehmen nicht mitmachen (Unternehmen D).“ Als zusätzlich erschwerend zeigte sich der Umstand, dass in zwei der beteiligten Unternehmen vorher nicht absehbare tiefgreifende organisationale Veränderungsprozesse (neue Geschäftsleitung und Nachfolgeregelungen) einen Grossteil der internen Ressourcen absorbierte. Auf diesem Hintergrund entschied sich das Forschungsteam für einen offenen und flexiblen Projektrahmen bei der Datensammlung für die Aussensicht.

Im Rahmen einer Fokusgruppe wurden die Projektleitenden in die Grundlagen des Stakeholder Managements eingeführt und in anschließenden Beratungsgesprächen wurden die Selektion und Priorisierung ebenso wie die Erhebungsmethodik mit jedem der Unternehmen diskutiert. Das Vorgehen im konkreten Auswahlprozess war denn auch sehr unterschiedlich. Während es für ein Unternehmen auf der Grundlage von früheren Erhebungen klar war, welches die relevanteste zu befragende Anspruchsgruppe ist, hat ein anderes Unternehmen einen anderen Weg gewählt. In einem moderierten Workshop mit 25 Mitarbeitern wurde eine Liste mit möglichen Anspruchsgruppen definiert, welche dann in intensiven Diskussionen auf die wichtigsten reduziert wurden. „Wir haben uns anschliessend auf fünf geeinigt, bei denen alle der Meinung waren, das sind unsere fünf wichtigsten Anspruchsgruppen (Unternehmen C)." Wenngleich das Vorgehen der Selektion und die gewählte Zahl der Stakeholder sehr unterschiedlich ausgefallen sind, unterstrichen doch alle Beteiligten im Experteninterview die folgenden Punkte mit einer erstaunlichen Übereinstimmung.

Der Einbezug der Stakeholder bei CSR-Themen wurde von allen befragten Unternehmen als sehr wichtig eingeschätzt. Es sei wirklich zu empfehlen, diesen Input abzuholen, damit die Aussicht in die Analyse einbezogen werden könne. ,Praktisch jeder, mit dem wir ein Interview geführt haben, hat das eigentlich sehr positiv aufgenommen und auch gesagt, das ist gut, dass ihr euch mal diesem Thema annehmt (Unternehmen C)." Die durchwegs positiven Rückmeldungen haben auch dazu geführt, dass sich die 
Tab. 2 Übersicht über die von den Unternehmen für die „Aussensicht“ ausgewählten Stakeholdergruppen und Methoden

\begin{tabular}{llll}
\hline Unternehmen & Stakeholdergruppen & Einbezogene Vertreter & Angewendete Methode \\
\hline A & Nichtregierungsorganisationen (NGO) & Vertreter einer entwicklungspolitischen, \\
unternehmenskritischen NGO & Moderierter Workshop \\
& Kunden des Detailhandels & Zwei Vertreter eines Kunden & Moderierter Workshop \\
B & Mitarbeitende & 31 Mitarbeitende & $\begin{array}{l}\text { Schriftliche Befragung, ergänzt } \\
\text { durch Einzelinterviews }\end{array}$ \\
& Kader & 9 Kaderangehörige & Schriftliche Befragung \\
C & Mitarbeitende & 25 Mitarbeitende & Online-Befragung \\
& Kunden & 19 Kunden & Einzelinterview \\
& Tourismusorganisationen & 3 Vertreter von 3 Organisationen & Einzelinterview \\
& Jugendliche/Schulen & 4 Leitungspersonen von 4 Schulen & Einzelinterview \\
& Gemeinden/Behörden & 6 Vertretende von 6 Gemeinden & Einzelinterview \\
& Kooperationspartner & 2 Vertretende von 2 Partnerunternehmen & Einzelinterview \\
D & Mitarbeitende & 12 Mitarbeitende & Moderierter Workshop \\
\hline
\end{tabular}

Unternehmen vorstellen können, auch nach dem laufenden Forschungsprojekt den Stakeholder-Dialog zu pflegen. „Der ganze Prozess an und für sich mit diesen Interviews und den Rückmeldungen an die Mitarbeiter nachher auch, das war schon sehr intensiv, aber es war gut zu sehen, dass das Ganze positiv aufgenommen wird und dass man das eigentlich noch mehr unterstützen sollte in Zukunft (Unternehmen B).“ Einige der befragten Projektleiter haben bereits beschlossen, die Mitarbeitenden beim Thema ,CSR“ auch weiterhin kontinuierlich und aktiv in die Diskussion einzubeziehen.

Ebenso wurde die Auswahl der zu befragenden Stakeholder als grosse Herausforderung übereinstimmend wahrgenommen. ,D Das ist eine ganz wichtige Frage, die man sich am Anfang stellen muss. Und ich glaube, man muss sich diese Frage wirklich gut stellen (Unternehmen A). “ Dass die fünf Unternehmen teilweise unterschiedliche Stakeholder priorisiert haben, wurde in der Fokusgruppe diskutiert. Dabei waren sich die Projektleiter einig, dass dies letztlich jedes Unternehmen für sich zu entschieden habe, wichtig sei nur ,,dass man wirklich die richtigen Stakeholder für sich herausfindet (Unternehmen B). " Konsens war, dass bei der Stakeholderauswahl standartisierte Vorgaben wenig Sinn machen. ,Wenn man ehrlich sein will, alle Stakeholder sind in diesem Thema nicht wichtig (Unternehmen D). “ Entscheidend waren beispielsweise Branche, lokale Verankerung, Wertschöpfungskette oder CSR-Fokus aus den ersten beiden Analyseschritten. In einer Frage aber waren sich alle einig: Die wichtigsten Stakeholder sind eindeutig die Mitarbeitenden im eigenen Betrieb. ,, Sie sind die wichtigsten Mitträger, die uns einerseits bei ISO 26000 unterstützen sollen, die wir aber damit auch gleichzeitig unterstützen wollen (Unternehmen B). " Die Mitarbeitenden wurden von den Projektleitern in den KMU in zweifacher Hinsicht priorisiert, als Träger für den Umsetzungsprozess von verantwortlichen Unternehmensaktivitäten und gleichzeitig als Zielgruppe welche von den Aktivitäten profitieren soll.

\section{Erkenntnisse zur Wahl der Methode für den Einbezug der Stakeholder}

Nach der Auswahl der zu befragenden Stakeholder wurde in einem nächsten Schritt die Frage der Methodenwahl zur Datenerhebung mit den einzelnen Projektleitenden fokussiert. Das Forschungsteam skizzierte die drei Formen moderierter Workshop, Fragebogen oder Einzelinterview als Möglichkeit für die Datenerhebung. Auf Unternehmensseite wurde in der Folge der Einsatz eines Fragebogens kontrovers diskutiert, was sich in den Experteninterviews deutlich zeigte. Als Vorteil nannten die befragten Projektleiter die Anonymität bei der Frageerhebung und der Einsatz bei einer größeren Personenanzahl. Als großer Nachteil wurde aber die hohe Komplexität des CSR-Themas und der Handlungsfelder bzw. Themenschwerpunkte von ISO 26000 aufgeführt. Mehrfach wurde betont, dass der Einsatz eines Fragebogens in diesem Bereich ,extrem schwierig“ sei und zu sprachlichen Missverständnissen führe. Das persönliche Gespräch mit den ausgewählten Stakeholdern, sei es in der Form von Einzelinterviews oder als moderierter Workshop, wurde denn auch von den Unternehmen gegenüber einer schriftlichen Befragung insgesamt deutlich bevorzugt (vgl. Tab. 2).

Die Wahl der ressourcenaufwändigen Methode mag erstaunen. In den Interviews wurde dies wie folgt begründet. Nur so könne herausgespürt werden, was die befragte Person sagen will, man erhalte genauere Informationen. „Ich erachte es als sehr wertvoll dass mir der Stakeholder direkt gegenüber sitzt. Erstens lerne ich die Person genauer kennen, dann sehe ich die Reaktion auf verschiedene Aspekte und dann ich Unklarheiten gleich in der Diskussion klären (Unternehmen A).“ In einem Beispiel wurden acht Mitarbeitende geschult und für rund 40 Interviews aus verschiedenen Anspruchsgruppen eingesetzt. Die Resultate wurden trotz des großen Aufwands durchwegs als positiv eingeschätzt, was zum einen die Bedeutung der persönlichen Beziehung 
im Stakeholderdialog für KMU unterstreicht. Zum anderen aber zeigt sich die bereits im Vorfeld geäußerte Skepsis gegenüber der Komplexität de Norm ISO 26000 auch in diesem Beispiel. Die Interviewer erzählten immer wieder vom Erklärungsbedarf für das Verständnis der einzelnen Handlungsfelder.

Im methodischen Vorgehen beim Einbezug der Mitarbeitenden wurde der partizipative und dialogische Prozess innerhalb des Unternehmens als Gewinn unterstrichen. „Der direkte Einbezug in Fragen bezüglich CSR wird von den Mitarbeitenden sehr geschätzt und sie merken auch, dass wir sie ernst nehmen. Das ist im psychologischen Bereich nicht zu unterschätzen (Unternehmen D).“ Eine wichtige Frage war auch, ob das Engagement der Unternehmensleitung intern gleich eingeschätzt werde oder ,sind wir mit unserer Chefetagenwahrnehmung komplett daneben? (Unternehmen B).“ Es sei wichtig zu sehen, dass die beiden Positionierungen hinsichtlich der Innensicht und der Aussensicht übereinstimmen würden. Für diejenigen Unternehmen mit einer "Teppichetage und Nichtteppichetage“ war es zentral die „Einschätzung der Mitarbeitenden auf Augenhöhe abzuholen und die Bedürfnisse auch zu spüren (Unternehmen B).“ Das Engagement wurde geschätzt und die Mitarbeitenden ,sind offensichtlich sehr stolz darauf, dass sie hier arbeiten (Unternehmen B).“

Trotz der durchwegs positiven Rückmeldungen in den Experteninterviews zu den Erfahrungen im Stakeholderdialog und den manchmal überraschenden und neuen Sichtweisen auf Seiten der Unternehmen bleiben viele Fragen der Implementierung eines Stakeholderdialogs für KMU offen. Ein Projektleiter brachte die methodischen Herausforderungen im gewählten Vorgehen am konkreten Beispiel auf den Punkt und löste mit seiner Aussage wichtige Diskussionen im Pilotprojekt im Hinblick auf die nächsten Entwicklungsschritte aus. „Was soll man tun, wenn ein Befragter ein Handlungsfeld als sehr wichtig einstuft und ein anderer aus derselben Anspruchsgruppe als unwichtig? Unternehmen C)." Eine Aggregation zu Durchschnittswerten verwischt die Unterschiede und ist bei der kleinen Stichprobe wenig zielführend. Aus Sicht des Forschungsteams werden hier die Grenzen des pragmatischen Vorgehens, der situativ angepassten Wahl qualitativer Methoden und der offenen Rahmenbedingungen deutlich.

\section{Fazit aus dem Einbezug der Stakeholder im Rahmen des Pilotprojekts}

Insgesamt wurde der Einbezug der Stakeholder in allen Experteninterviews als sehr positiv beurteilt. Die persönlichen Gespräche und das Kennenlernen der unterschiedlichen Stakeholder und deren Bedürfnisse und Anliegen wurden sowohl von Seiten der Unternehmen, wie auch von Seiten der befragten Stakeholder geschätzt und als wert- voll empfunden. Der Gewinn eines vertieften Austausches wurde dabei betont: ,Solche Sachen werden einem erst wirklich bewusst in dem Moment, wenn man das mit den Leuten direkt anspricht und nachhaken kann, warum bestimmte Themen für die Anspruchsgruppe wichtig sind. Dadurch ist es auch möglich, die Hintergründe zu verstehen warum ein bestimmtes Thema als sehr wichtig eingeschätzt wird (Unternehmen B)." Durchgehend wurde betont, dass die Sicht von unterschiedlichen Stakeholdern als wesentlicher Input für den weiteren Entscheidungsprozess verstanden wurde, ,zum Sagen wo können, wo wollen wir ansetzen als Firma? Wo sehen wir Handlungsfelder, wo wir uns eigentlich verbessern wollen (Unternehmen A)." Aber nicht nur zukunftsgerichtet, auch im Hinblick auf die Außenwahrnehmung wurden positive Effekte genannt. ,Jetzt wissen wir besser, ob wir eigentlich das Richtige tun oder ob es irgendwo Bereiche gibt wo wir noch nicht aktiv sind. Das hat man aus den Interviews gut herausgespürt (Unternehmen C).“

Für das Forschungsteam stellen sich im Hinblick auf das weitere Vorgehen wichtige Fragen zum Einbezug der Stakeholder durch KMU bei der Anwendung von ISO 26000. Die skizzierte Auswahl der Stakeholder und die methodische Datenerhebung sind ein erstes wichtiges Lernfeld bezüglich der nächsten Entwicklungsaufgaben. Teilweise wurde die Stakeholderbefragung nur für interne Stakeholder durchgeführt, obwohl auch die Befragung externer Stakeholder wünschbar gewesen wäre. Als wichtiger Grund wurde von den Unternehmen der zeitliche Aufwand angeführt. Bei den befragten Projektleitern zeigte sich im Weiteren aber auch dass kaum Erfahrungen mit einem systematischen externen Stakeholder Dialog bei KMU vorliegen. Wenngleich hier unterstützende Instrumente und Beratungsleistungen anzudenken sind, zeigen die Experteninterviews doch deutlich, dass vor einem allzu wissenschaftlichen und formalistischen Vorgehen abzusehen ist. Es scheint für KMU wichtig zu sein, mit einer Offenheit für qualitative und pragmatische Ansätze einen ersten Schritt in Richtung Stakeholderdia$\log$ zu machen. Im Hinblick auf das Prozesshandbuch sind darum folgende Hilfestellungen vorzusehen:

- Empfehlung der Auswahl von drei Anspruchsgruppen für die Stakeholderbefragung (davon zwei externe);

- Entwicklung von Checklisten für Stakeholderauswahl;

- Entwicklung von einfachen Tools zur Stakeholderbefragung.

Als zweites wichtiges Entwicklungsfeld ist die Komplexität der CSR-Thematik bzw. der anspruchsvolle Rahmen von ISO 26000 mit seinem Anspruch auf globale Gültigkeit festzuhalten. Die Stakeholderbefragung wurde von einigen Unternehmen auf die Kernthemen reduziert, da eine Analyse nach Handlungsfeldern als zu umfangreich und aufwändig erschien. In aller Regel mussten die Kernthemen und Handlungsfelder zum besseren Verständnis mündlich vor oder 
während der Befragung erläutert und kommentiert werden. Um ISO 26000 auch für KMU anwendbar zu machen, liegt hier eine große Herausforderung. Es gilt Vereinfachungen, Übersetzungen mit Beispielen vielleicht auch Visualisierungen zu finden, welche den Kern der Handlungsfelder im Kontext eines mitteleuropäischen KMU treffen ohne durch die Reduktion an Inhalt zu verlieren. Dieses Spannungsfeld wird nicht einfach zu lösen sein. Folgende Hilfsmittel stehen für die Weiterentwicklung im Zentrum:

- Angepasste Instrumente für die Stakeholderbefragung, ausdifferenziert nach den Vorkenntnissen der Stakeholdergruppen;

- Ein Katalog von regions- und branchenspezifischen Beispielen je Handlungsfeld;

- Eine Checkliste für die Kommunikation, ausdifferenziert nach Stakeholdergruppe.

Mit den geplanten Hilfestellungen im Rahmen eines Prozesshandbuchs können Grundlagen geschaffen werden, damit KMU einen ersten Schritt in Richtung des Einbezugs von Stakeholdern bei der Beurteilung der Handlungsfelder machen können. Für die Implementierung von ISO 26000 ist das ein wesentliches Element.

\section{Dank}

Ein besonderer Dank gilt Clemens Lang und Alex Kunze für ihre maßgebliche Mitarbeit bei der Konzeption und Realisierung des Pilotprojekts sowie Angelika Hitthaler und Fabrizio Marra für ihre Unterstützung bei der Führung und Auswertung der Interviews.

\section{Literatur}

Burchell J, Cook J. (2006) Assessing the impact of stakeholder dialogue: changing relationships between NGOs and companies. J Public Aff 6:210-227

Camillus JC (2008) Strategy as a wicked problem. Harvard Bus Rev 86(5):99-106

Castka P, Balzarova MA, Bamber CJ, Sharp JM (2004) How can SMEs effectively implement the CSR Agenda? A UK case study perspective. Corporate Soc Respon Environ Manage 11:140-149

Crane A, Livesey S (2003) Are you talking to me? Stakeholder communication and the risks and rewards of dialogue. In: Andriof J, Waddock S, Husted B (Hrsg) Unfolding stakeholder thinking, Bd 2. Greenleaf Publishing Limited, Sheffield, p 39-52

Daub CH, Stiller Y, Stiller S, Ergenzinger R, Scherrer Y (2007) Die besten Geschäftsberichte der Schweiz -- Aktuelle Ergebnisse und Bilanz einer Studie über fünf Jahre. Edition Gesowip, Basel

Eichholz V (2007) Gesellschaftliches Engagement in kleinen und mittelständischen Unternehmen in Deutschland - aktueller Stand und zukünftige Entwicklung. Studie erstellt durch die GILDE GmbH im Auftrag der EU-Kommission. Gilde, Detmold
Enderle G (2004) Global competition and corporate responsibilities of small and medium-sized enterprises. Bus Ethics Eur Rev 13(1):51-63

Europäische Kommission (Hrsg) (2001) Grünbuch - Europäische Rahmenbedingungen für die soziale Verantwortung der Unternehmen. KOM(2001) 366. Brüssel

Europäische Kommission (Hrsg) (2011) Eine neue EU-Strategie (2011-2014) für die soziale Verantwortung der Unternehmen (CSR). KOM(2011) 681. Brüssel

Freeman RE, Harrison JS, Wicks A (2007) Managing for stakeholders. Survival, reputation, and success. Yale University Press, New Haven, London

GRI (2011) Sustainability Reporting Guidelines, Version 3.1. Global Reporting Initiative, Amsterdam

Hardtke A, Kleinfeld A (Hrsg) (2010) Gesellschaftliche Verantwortung von Unternehmen. Von der Idee der Corporate Social Responsibility zur erfolgreichen Umsetzung. Gabler, Wiesbaden

Jakob MC, von Passavant C (2009) Corporate social responsbility. Impulse für KMU. Huber, Frauenfeld

Jamali D, Zanhour M, Keshishian T (2009) Peculiar strengths and relational attributes of SMEs in the context of CSR. J Bus Ethics $87: 355-377$

Jenkins H (2004) A critique of conventional CSR theory: an SME perspective. J Gen Manage 29(4 Summer):37-57

Klink D (2008) Der Ehrbare Kaufmann - Das ursprüngliche Leitbild der Betriebswirtschaftslehre und individuelle Grundlage für die CSR-Forschung. Z Betriebswirtschaft 2008(3):57-79

Lang C, Kunze A, Jakob MC, Winistörfer H (2012) ISO 26000 nutzen - Wegweisendes Projekt für Schweizer KMU. In: Management und Qualität 12/2012:24-25

Lang C, Winistörfer H (2010) Wie gesellschaftliche Verantwortung international umgesetzt werden kann. Der Standard ISO 26000 erleichtert den weltweiten Vergleich, wie Unternehmen ihre soziale Verantwortung wahrnehmen. In: io new management 5/2010:24-27

Lepoutre J, Heene A (2006) Investigating the impact of firm size on small business responsibility: a critical review. J Bus Ethics 67:257-273

Murillo D, Lozano J (2006) SMEs and CSR: an approach to CSR in their own words. J Bus Ethics 67:227-240

Perrini F, Minoja M (2008) Strategizing corporate social responsibility: evidence from a Italian medium-sized, family-owned company. Bus Ethics Eur Rev 17(1):47-63

Sachs S, Rühli E, Kern I (2007) Lizenz zum Managen. Mit Stakeholdern zum Erfolg: Herausforderungen und Good Practices. Haupt, Bern

SNV (2011) SNR ISO26000. Leitfaden zur gesellschaftlichen Verantwortung. SNV Schweizerische Normen-Vereinigung, Winterthur

Spence L (1999) Does size matter? The state of the art in small business ethics. Bus Ethics Eur Rev 8(9):163-172

Spence L, Schmidpeter R, Habisch A (2003) Assessing social capital: small and medium sized entreprises in Germany and the UK. J Bus Ethics 47:17-29

Vyakarnam S, Bailey A, Myers A, Burnett D (1997) Towards an understanding of ethical behaviour in small firms. J Bus Ethics 16(15):1625-1636

Winistörfer H, Perrin I, Teuscher P, Forel A (2012) Management der sozialen Verantwortung in Unternehmen. Leitfaden zur Umsetzung. Hanser, München

Yaziji M, Doh JP (2009) NGOs and corporations. Conflict and collaboration. Cambridge University Press, Cambridge 